\title{
The Current Status of Bird Diversity in Yunnan Province
}

\author{
YANG LAN WEN XIANJI YANG XIAOJUN \\ Kunming Institute of Zoology, the Chinese Academy of Sciences, Kunming, Yunnan 650223 \\ (Received June 15, 1994; Revised February 28, 1995)
}

\section{ABSTRACT}

Yunnan is the richest province in bird species in China. Seven hundred and ninety-two species of birds were recorded in Yunnan before 1994, accounted for 63.6\% of the records in China. Among them, one hundred and twenty-seven species were very rare, two hundred and eighty-eight species were rare, two hundred and fourty-two species were common and thirty-five species were large in number. In China, Podargidae and Hemiprocnidae were only recorded in Yunnan, Trogonidae, Bucerotidae, Eurylaimidae, Pittidae, Campephagidae, Irenidae, Dicruridae, Timaliinae, Dicaeidae and Nectariniidae mainly distributed in Yunnan, and one hundred and twelve species were only recorded from Yunnan. One hundred and thirty-six species belonged to the nation's prior protected wildlife. Their status in Yunnan were: five species extinct, seven species endangered, five species vulnerable, ninety-eight species rare and twenty-one species common. Twenty-five species were listed in 1988 IUCN Red List of Threatened Animals. Anser anser and A. indicus were the province's prior protected birds. Thirty nature reserves have played an important role in protecting birds and their habitats, especially Xishuangbanna, Gaoligongshan, Ailaoshan and Wuliangshan. The public education about the protection of birds and their habitats has been made universal since 1982. It is important to protect their habitats both inside and outside nature reserves and recover of destroyed habitats. Illegal hunting and capturing must be prohibited, breeding of economic birds in captivity should be started, and the education concerning the protection of birds and their habitats must be continued.

Key words bird diversity, conservation, protected birds, status, Yunnan

\section{Introduction}

Yunnan Province, which is located in southwest of China $\left(21^{\circ} 08^{\prime} \sim 29^{\circ} 08^{\prime} \mathrm{N}, 97^{\circ} 31^{\prime} \sim\right.$ $106^{\circ} 12^{\prime} \mathrm{E}$ ), is geographically the main part of Yunnan-Guizhou Plateau. Its total area is about 383000 square kilometers. About $94 \%$ of the total area are mountainous areas. It slopes downward from northwest to southeast. The highest point is Kagebo Peak of Meli Snow Mountain, at an elevation of $6740 \mathrm{~m}$, in the northwest of Yunnan. The lowest point is $76.4 \mathrm{~m}$ above sea level in Yunjiang (Red River) outlet of southeast of Yunnan.

Yunnan Province is within the zone of monsoon climate in tropics and subtropics. The complex topography endows this Province with climate differences horizontally and vertically. The composite climate of frigid, temperate and torrid zones can be found here. Vegetation is mainly composed of tropical rain forest, monsoon forest, subtropical evergreen broadleafed forest and subalpine coniferous forest. Tropical rain forest and monsoon forest are in southern part at low altitude and elevation. Subtropical evergreen broad-leaf forest scatters in the most of mountainous parts of the Province. Pine forest, shrubs or grassland begin to 
grow where broadleaf used to after destruction. Subalpine coniferous forest is found in high mountain in northwestern part. The complex topography, climate and vegetation in Yunnan Province provide various ideal habitats for birds.

\section{Species diversity and current status}

Seven hundred and ninety-two species of birds, which were recorded in Yunnan before 1994, belonged to 19 orders, 68 families ( 4 subfamilies). Compared with other provinces (autonomous regions) in China, Yunnan is the richest province in birds. Table 1 showed the results of the comparison of bird species between Yunnan and its adjacent provinces.

Table 1 Illustrates bird species in China, Yunnan and its adjacent provinces

\begin{tabular}{lccccl}
\hline Regions & Orders & Families & Species & Percentage $^{*}$ & Resource \\
\hline China & 21 & 82 & $1244(2 ?)$ & 100 & Cheng Tso-hsin, 1994 \\
Yunnan & 19 & 69 & $792(4 ?)$ & 63.6 & Authors \\
Sichuan & 19 & 59 & 571 & 45.9 & Shi Bainan et al., 1984 \\
Guizhou & 17 & 51 & 403 & 32.4 & Wu Zhikang et al. , 1986 \\
Guangxi & 19 & 56 & 496 & 39.8 & WAE, 1985 \\
Xizarig(Tibet) & 19 & 57 & 473 & 38.0 & CSEQXP, 1983 \\
\hline
\end{tabular}

* Of total species in China

In China, Podargidae and Hemiprocnidae were only recorded in Yunnan, Trogonidae, Bucerotidae, Eurylaimidae, Pittidae, Campephagidae, Irenidae, Dicruridae, Timaliinae, Dicaeidae and Nectariniidae mainly distributed in Yunnan, and one hundred and twelve species were only recorded from Yunnan.

Based on the data from the field work and data from some publications, the status of seven hundred and ninety-two species in Yunnan can be roughly divided into very rare, rare, common and dominant species. Table 2 showed the results.

Table 2 The number of species and percentage of their different status

\begin{tabular}{lcccc}
\hline Status & Very rare & Rare & Common & Dominant \\
\hline Number of species & 127 & 288 & 342 & 35 \\
Percentage (\%) & 16.04 & 36.36 & 43.18 & 4.42 \\
\hline
\end{tabular}

\section{The protected birds and their status}

Among the seven hundred and ninety-two species of birds, eighteen species belonged to the nation's prior first-ranked protected birds, accounting for $49 \%$ of those in China. One hundred and eighteen species belonged to the second-ranked protected birds, accounting for $71 \%$ of total second-ranked protected birds in China. Twenty-five species were listed in 1988 IUCN Red List of Threaten Animals. The protected birds, their important levels in protection in China, status and distribution in Yunnan were listed in appendix.

Among the protected birds recorded in Yunnan, Spot-billed Pelican (Pelecanus philippensis), Painted Stork (Ibis leucocephalus), Sacred Ibis (Threskiornis aethiopicus), Black Ibis (Pseudibis papillosa) and Malaya Fish-Owl (Ketupa ketupu) were not found over past 50 years. It seemed that they were extinct in Yunnan. Seven species such as Spoonbill 
(Platalea leucorodia), Black Vulture (Sarcogyps calvus) and Sarus Crane (Grus antigone) were common at the end of $1950 \mathrm{~s}$ and the beginning of $1960 \mathrm{~s}$, but it has been very difficult to see them ever since then. They have become the endangered birds in Yunnan. Five species were vulnerable, accounting for $3.68 \%$ of total protected birds in Yunnan, ninety-eight species were rare, accounting for $72.06 \%$, twenty-one species were common, accounting for $15.44 \%$.

In addition to all these nation's prior protected birds, Greylag Goose (Anser anser) and Bar-headed Goose ( $A$. indicus) were the province's prior protected birds because of their small population in Yunnan. Crypsirina temnura, Brachypteryx hyperythra, Garrulax maesi, G. galbanus, G. lunulatus, G. formosus, Paradoxornis flavirostris, P. ruficeps, Sitta magna, S. formosa, and S. yunnanensis, which were not listed as protected birds in China, were listed in 1988 IUCN Red List of Threaten Animals. Among them, Crypsirina temnura and Garrulax formosus were vulnerable, Garrulax lunulatus and Paradoxornis flavirostris were common, all others were rare.

\section{Conservation and recommendations}

The main reason for the reduction of bird diversity and the decreasing in number of many species was due to the destruction of their habitats. When forest was cut down and burnt, and wetlands were drained and polluted, many forest birds disappeared or their population decreased rapidly in Yunnan because of the loss of their habitats. Since 1980, 30 nature reserves have been reestablished in Yunnan, and they have played a very important role in protecting birds. Among them, Xishuangbanna, Gaoligongshan, Ailaoshan and Wuliangshan Nature Reserves are the most important reserves because of their large size and unique location (Table 3).

Table 3 The most important reserves in Yunnan Province

\begin{tabular}{|c|c|c|c|c|c|c|}
\hline \multirow{2}{*}{ Reserves } & \multirow{2}{*}{$\operatorname{Size}^{*}\left(\mathrm{hm}^{2}\right)$} & \multicolumn{2}{|c|}{ Species } & \multicolumn{2}{|c|}{ Protected birds } & \multirow{2}{*}{ Literature } \\
\hline & & Number & $\% *$ & Number & $\% * *$ & \\
\hline Xishuangbanna & 241776 & 427 & 53.9 & 79 & 58.1 & Yang Yuangchang et al., 1987 \\
\hline Gaoligongshan & 123900 & 343 & 43.1 & 38 & 27.9 & Author \\
\hline Ailaoshan & 50360 & 323 & 40.8 & 13 & 9.6 & Wei Tianhao et al., 1988 \\
\hline Wuliangshan & 23355 & 290 & 36.3 & 30 & 22.5 & Author \\
\hline
\end{tabular}

* From FSPIYP, 1989; **: Of the total species in Yunnan Province; ***: Of the total protected birds in Yunnan Province

Yunnan is a province consisted of many minority nationalities. Most of them like hunting, which is another threat to birds. Hundreds and thousands of partridges, pheasants, ducks and doves were killed each year for their meat, beautiful plumage or down feather. Among thirty-two Galliformes birds in Yunnan, five species were very rare, seventeen species were rare, eight species were common and only two species were large in number. Among twenty-five Anseriformes birds, four species were very rare, nine species were rare, eight species were common and four species were large in number. Because of over-hunting, the population of many species, especially the economic species, were decreasing rapidly. From 1954 to 1963 purchasing of pheasant skins (main Ring-necked, Lady Amherst's and Silver Pheasant) in Yunnan was 32867 yearly, which was much more than that from 1964 to 
1984. From 1964 to 1984 the yearly average of purchasing of pheasant skins was 14057 . The decrease rate was 703 skins per year. Every year hundreds and thousands of traditional ornamental birds such as Red-breasted parakeet, Gray-headed parakeet, Hill Myna, Whitevented Myna, Hwamei, Silver-eared Leiothrix, Red-billed Leiothrix, Rosefinchs also were captured and sold in markets.

Since 1982, "Love Birds Week" in every spring was nation-wide inaugurated. Every year from April 1 to April 7 it is the "Love Birds Week" in Yunnan Province. During this week the citizens were educated by posters, newspapers, radio and TV. Competitions of birds watching among middle-school students and competitions of the knowledge about protecting birds and their habitats among citizens were held from 1986 to 1988 . Most people in cities and towns have come to understand the importance of protecting birds and their habitats. On November 8, 1988, the Chinese Wildlife Protection Law was adopted by National People's Congress. With that people began to know it was illegal to hunt and trade wildlife, but the illegal hunting and trading of wildlife still happened occasionally, especially in the remote countryside.

Most species occurred in thirty nature reserves. Some protected birds now can be found only in nature reserves. These reserves have taken an important role in protecting birds. However, their sizes were too small to protect all birds. So, the protection of bird habitats outside nature reserves and the recovery of their destructed habitats are also important. To breed economic birds in captivity should be started so that the hunting and capturing pressure in field can be decreased. The education concerning the protection of birds and their habitats must be continued also in order to protect birds.

\section{REFERENCES}

Cheng Tsohsin, 1994. A complete checklist of species and subspecies of the Chinese birds. Beijing: Science Press, $1-318$ (in Chinese)

CSEQXP (The Comprehensive Scientific Expedition to Qinghai-Xizang Plateau), Academia Sinica, 1983. The avifauna of Xizang. Beijing: Science Press, 3 37 (in Chinese)

FSPIYP (Forestry Surveying and Planning Institute of Yunnan Province), 1989. The nature reserves in Yunnan. Beijing: China Forestry Publishing House, $31-32$ (in Chinese)

Shi Bainan, Zhao Ermi, 1982. Sichuan fauna economica. Chengdu: Sichuan People's Publishing House, 1 : 96- 145 (in Chinese)

The IUCN Conservation Monitoring Centre, 1988. 1988 IUCN Red List of Threatened Animals. IUCN, Gland, Switzerland, $21 \sim 57$

WAE (Wild Animal Expedition, Forestry Surveying and Designing Institute of Guangxi Autonomous Region), 1985. The distributional list of wild animals in Guangxi. $1 \sim 89$ (in Chinese)

Wei Tianhao, Wang Zhijun, 1988. The birds in northern and middle parts of the Ailaoshan Mountains. In: Comprehensive Expedition to Ailaoshan Nature Reserve (ed. ), The reports of comprehensive expedition to Ailaoshan Nature Reserve, Kunming: Yunnan Nationality Press, 206 -230 (in Chinese)

Wu Zhikang et al., 1986. The avifauna of Guizhou. Guiyang: Guizhou People's Publishing House (in Chinese)

Yang Yuanchang et al., 1987. On birds from Xishuangbanna. In: Comprehensive Expedition to Xishuangbanna Nature Reserve (ed.), The reports of comprehensive expedition to Xishuangbanna Nature Reserve. Kunming: Yunnan Scientific and Technological press, 326 349 (in Chinese) 
Appendix The distribution and status of protected birds in Yunnan Province

\begin{tabular}{|c|c|c|c|c|}
\hline \multicolumn{2}{|c|}{ Names } & \multirow{2}{*}{$\frac{\text { ILP }}{2 \text { nd }}$} & \multirow{2}{*}{$\frac{\text { Status }}{E x}$} & \multirow[t]{2}{*}{ Distribution record } \\
\hline 1 & Pelecanus philippensis & & & \\
\hline 2 & Phalacrocorax niger & 2nd & $\mathbf{R}$ & Dehong, Lincang, Southern Yunnan \\
\hline 3 & Ibis leucocephalus & 2nd & Ex & Honghe \\
\hline 4 & Ciconia nigra & 1st & $\mathbf{R}$ & $\begin{array}{l}\text { Diqing, Lijiang, Dali, Baoshan, Kunming, } \\
\text { Qujing, Honghe }\end{array}$ \\
\hline 5 & Threskiornis aethiopicus & 2nd & Ex & Honghe \\
\hline 6 & Psendibis papillosa & 2nd & Ex & Southwestem Yunnan \\
\hline 7 & Platalea leucorodia & 2nd & $E$ & Kunming, Qujing \\
\hline 8 & Cygnus cygnus & 2nd & $\mathrm{V}$ & Diqing \\
\hline 9 & Aix salericulata & 2nd & $\mathbf{R}$ & Kunming, Zhaotong, Quijing, Lincang, Chuxiong \\
\hline 10 & Elanus caeruleus & 2nd & $\mathbf{R}$ & $\begin{array}{l}\text { Kunming. Qujing, Yuxi, Baoshan, Dali, Wenshan, Xishuangbanna, } \\
\text { Simao }\end{array}$ \\
\hline 11 & Aviceda jerdoni & 2nd & $\mathbf{R}$ & Lincang, Xishuangbanna \\
\hline 12 & A. leuphotes & 2nd & $\mathrm{R}$ & Honghe, Xishuangbanna \\
\hline 13 & Pernis ptilorhynchus & 2nd & $\mathbf{R}$ & $\begin{array}{l}\text { Kunming, Honghe, Lincang, Baoshan, Lijiang, Xishuangbanna, } \\
\text { Chuxiong }\end{array}$ \\
\hline 14 & Milvus migrans & 2nd & $C$ & $\begin{array}{l}\text { Kunming, Baoshan, Lijiang, Xishuangbanna, Lincang, Dehong, } \\
\text { Zhaotong }\end{array}$ \\
\hline 15 & Haliast ur indus & 2nd & $\mathbf{R}$ & Dehong, Gaoligongshan, Lijiang, Simao \\
\hline 16 & Accipiter gentilis & 2nd & $\mathbf{R}$ & Kunming, Zhaotong, Lijiang, Honghe, Xishuangbanna \\
\hline 17 & A. badius & 2nd & $\mathbf{R}$ & Xishuangbanna, Baoshan, Gaoligongshan \\
\hline 18 & A. trivirgatus & 2nd & $\mathbf{R}$ & $\begin{array}{l}\text { Kunming, Qujing, Simao, Honghe, Baoshan, Gaoligongshan, } \\
\text { Xishuangbanna }\end{array}$ \\
\hline 19 & A. nisus & 2nd & $\mathrm{C}$ & $\begin{array}{l}\text { Xishuangbanna, Simao, Lincang, Nujiang, Kunming, } \\
\text { Zhaotong }\end{array}$ \\
\hline 20 & A. virgatus & 2nd & C & All parts of Yunnan \\
\hline 21 & Buteo rufinus & 2nd & $\mathbf{R}$ & Kunming \\
\hline 22 & B. hemilasius & 2nd & $\mathbf{R}$ & Kunming \\
\hline 23 & B. buteo & 2nd & $\mathrm{C}$ & All parts of Yunnan \\
\hline 24 & B. lagopus & 2nd & $\mathbf{R}$ & Lijiang \\
\hline 25 & Butastur indicus & 2nd & $\mathbf{R}$ & Xishuangbanna, Honghe \\
\hline 26 & B. liventer & 2nd & $\mathbf{R}$ & Xishuangbanna, Simao \\
\hline 27 & Spizaetus nipalensis & 2nd & $\mathbf{R}$ & Lijiang, Weixi \\
\hline 28 & S. cirrhatus & 2nd & $\mathbf{R}$ & Xishuangbanna \\
\hline 29 & Aquila chrysaetos & 1 st & $\mathbf{R}$ & Kunming, Baoshan, Diqing \\
\hline 30 & A. heliaca & 1 st & $\mathbf{R}$ & Dehong \\
\hline 31 & A. rapax & 2nd & $\mathbf{R}$ & Kunming, Yuxi, Lijiang, Honghe, Xishuangbanna \\
\hline 32 & A. fasciata & 2nd & $\mathbf{R}$ & Kunming \\
\hline 33 & A. pennata & 2nd & $\mathbf{R}$ & Xishuangbanna \\
\hline 34 & A. kienerii & 2nd & $\mathbf{R}$ & Dehong \\
\hline 35 & Ictinaetus malayensis & 2nd & $\mathbf{R}$ & Xishuangbanna, Baoshan, Gaoligongshan \\
\hline 36 & Sarcogyps calvus & 2nd & $\mathrm{E}$ & Dehong, Xixhuangbanna, Simao \\
\hline 37 & Aegvpius monachus & 2nd & $E$ & Southern Yunnan \\
\hline 38 & Gyps himalayensis & 2nd & $\mathbf{R}$ & Gaoligongshan, Nushan, Zhaotong \\
\hline 39 & $G$. bengalensis & 1st & $\mathrm{E}$ & Xishuangbanna, Dehong \\
\hline 40 & Gypaetus barbatus & $1 \mathrm{st}$ & $\mathbf{R}$ & Northwestern Yunnan \\
\hline 41 & Circus cyaneus & 2nd & $\mathbf{R}$ & Kunming, Qujing, Yuxi, Zhotong, Dali, Baoshan \\
\hline 42 & C. melanoleucos & 2nd & $\mathrm{C}$ & Kunming, Honghe, Simao, Dehong, Xishuangbanna, Lincang \\
\hline 43 & C. aeruginosus & 2nd & $\mathbf{R}$ & Kunming, Yuxi \\
\hline 44 & C. spilonotus & 2nd & $\mathbf{R}$ & Kunming, Honghe, Xishuangbanna \\
\hline 45 & Spilornis cheela & 2nd & $\mathbf{R}$ & Dehong, Baoshan, Lincang, Honghe, Xishuangbanna \\
\hline 46 & Pandion haliaetus & 2nd & $\mathbf{E}$ & Honghe \\
\hline 47 & Microhierax caerulescens & 2nd & $\mathbf{R}$ & Dehong \\
\hline & M. melanoleucos & 2nd & $\mathbf{R}$ & Xishuangbanna, Lincang, Honghe, Wenshan, Zhaotong \\
\hline
\end{tabular}




\begin{tabular}{|c|c|c|c|c|}
\hline 49 & Falco peregrinus & 2nd & $\mathrm{R}$ & Kunming, Xishuangbanna \\
\hline 50 & F. subbuteo & 2nd & $\mathrm{R}$ & Kunming, Baoshan, Dehong, Lijiang \\
\hline 51 & $F$. severus & 2nd & $\mathrm{R}$ & Dehong, Simao, Xishuangbanna \\
\hline 52 & F. columbarius & 2nd & $\mathbf{R}$ & Dehong \\
\hline 53 & $F$. vespertinus & $2 n d$ & $\mathbf{R}$ & Xishuangbanna \\
\hline 54 & F. naumanni & 2nd & $\mathbf{R}$ & Qujing, Western Yunnan \\
\hline 55 & F. tinnunculus & 2 nd & $\mathrm{C}$ & All parts of Yunnan \\
\hline 56 & Tetrastes serverzowi & $1 \mathrm{st}$ & $\mathrm{R}$ & Diqing \\
\hline 57 & Tetraogallus tibetanus & 2 nd & $\mathrm{R}$ & Diqing \\
\hline 58 & Tetraophasis obscurus & $1 \mathrm{st}$ & $\mathrm{R}$ & Lijiang, Diq̨ing \\
\hline 59 & Ithaginis cruentus & 2nd & $\mathbf{R}$ & Northwestern Yunnan \\
\hline 60 & Tragopan satyra & $1 \mathrm{st}$ & $\mathbf{R}$ & Gaoligongshan \\
\hline 61 & T. blythii & $1 \mathrm{st}$ & $\mathbf{R}$ & Gaoligongshan \\
\hline 62 & T. temminckii & 2nd & $c$ & Baoshan, Nujıang, Lijiang, Diqing, Jingdong, Xinping \\
\hline 63 & Lophophorus sclateri & $1 \mathrm{st}$ & $\mathbf{R}$ & Gaoligongshan, Nushan \\
\hline 64 & L. Lhreysii & 1 st & $\mathrm{R}$ & Zhongdian \\
\hline 65 & Crossoptilon crossoptilon & 2 nd & $\mathrm{R}$ & Lijiang, Dıqing \\
\hline 66 & Lophura leucomelana & 2nd & $\mathrm{R}$ & Nujiang \\
\hline 67 & L. nycthemera & 2nd & $\mathrm{C}$ & $\begin{array}{l}\text { Dehong. Baoshan, Lincang, Simao, Xishuangbanna, Honghe, Wen- } \\
\text { shan, Zhaotong }\end{array}$ \\
\hline 68 & Gallus gallus & 2 nd & $\mathrm{C}$ & Dehong, Lincang, Simao, Honghe, Xishuangbanna, Wenshan \\
\hline 69 & Pucrasia macrolopha & 2nd & $\mathbf{R}$ & Lijiang, Nujiang, Diqing \\
\hline 70 & Syrmaticus humiae & 1 st & $\mathbf{R}$ & $\begin{array}{l}\text { Dehong, Baoshan, Lincang, Simao, Chuxiong, Dali, Lijiang, } \\
\text { Diqing }\end{array}$ \\
\hline 71 & S. reevesii & $1 \mathrm{st}$ & $\mathrm{V}$ & Zhaotong \\
\hline 72 & Chrysolophus amherstiae & 2 nd & $\mathrm{C}$ & Most parts of Yunnan \\
\hline 73 & C. pictus & 2 nd & $\mathbf{R}$ & Zhaotong \\
\hline 74 & Polyplectron bicalcaratum & 1 st & $\mathrm{R}$ & Dehong, Xishuangbanna \\
\hline 75 & Pavo muticus & $1 \mathrm{st}$ & $\mathrm{R}$ & $\begin{array}{l}\text { Dehong, Baoshan, Lincang, Dali, Chuxiong, } \\
\text { Honghe, Xishuangbanna }\end{array}$ \\
\hline 76 & Grus grus & $2 n d$ & C & $\begin{array}{l}\text { Kunming, Qujin;, Honghe, Diqing, Xishuangbanna, Lijiang, Zhao- } \\
\text { tong }\end{array}$ \\
\hline 77 & G. nigricollis & $1 \mathrm{st}$ & $\mathrm{R}$ & Kunming, Diqing, Zhongdian, Lijiang, Zhaotong, Qujing \\
\hline 78 & G. japonensis & $1 \mathrm{st}$ & $\mathrm{R}$ & Zhaotong \\
\hline 79 & G. antigone & $1 \mathrm{st}$ & $E$ & Xishuangbanna, Dehong, Gaoligongshan, Lincang, Zhongdian \\
\hline 80 & Porzana bicolor & 2nd & $\mathrm{R}$ & Lijiang, Yuxi \\
\hline 81 & Metopidius indicus & 2nd & $\mathrm{v}$ & Xishuangbanna \\
\hline 82 & Glareola lactea & 2nd & $\mathrm{V}$ & Honghe, Xishuangbanna, Dehong \\
\hline 83 & Sterna aurantia & 2nd & $\mathbf{R}$ & Xishuangbanna, Dehong \\
\hline 84 & Treron apicauda & $2 n d$ & $\mathbf{R}$ & Kunming, Xishuangbanna, Lincang, Dehong \\
\hline 85 & T. sphenura & 2nd & $\mathrm{C}$ & $\begin{array}{l}\text { Kunming, Yuxi, Simao, Honghe, Xishuangbanna, Lincang, } \\
\text { Dehong, Baoshan, Dali, Nujiang, Lijiang, Zhaotong }\end{array}$ \\
\hline 86 & T. phoenicoptera & 2nd & $\mathbf{R}$ & Xishuangbanna, Lincang, Dehong, Simao \\
\hline 87 & $T$. curvirostra & 2nd & $\mathrm{R}$ & Xishuangbanna, Simao, Yuxi, Baoshan \\
\hline 88 & T. pompadora & 2nd & $\mathbf{R}$ & Xishuangbanna \\
\hline 89 & Ducula aenea & 2nd & $\mathrm{R}$ & Xishuangbanna, Simao \\
\hline 90 & D. badia & 2nd & $\mathrm{R}$ & Xishuangbanna, Simao, Lincang, Dehong \\
\hline 91 & Macropygia unchall & 2nd & $\mathrm{R}$ & Xishuangbanna, Lincang, Dehong, Wenshan \\
\hline 92 & M. ruficeps & 2nd & $\mathrm{R}$ & Xishuangbanna \\
\hline 93 & Psittacula alexandri & 2nd & $\mathrm{R}$ & $\begin{array}{l}\text { Yuxi, Simao, Lincang, Baoshar, Dehong, Honghe, } \\
\text { Xishuangbanna }\end{array}$ \\
\hline 94 & P. derbiana & 2nd & $\mathrm{R}$ & Kunming, Simao, Lincang, Baoshan, Lijiang, Diqing \\
\hline 95 & P. himalayana & 2nd & $\mathrm{C}$ & $\begin{array}{l}\text { Ku:iming, Yuxi, Simao, Lincang, Baoshan, Dehong, Xishuangbanna, } \\
\text { Honghe, Dali, Nujiang }\end{array}$ \\
\hline 96 & Loriculus vernalis & 2nd & $\mathrm{v}$ & Simas \\
\hline 97 & Centropus sinensis & 2nd & $\mathrm{C}$ & Dehong, Xishuangbanna, Simao, Lincang, Honghe, Wenshan \\
\hline
\end{tabular}


Appendix (Continued)

\begin{tabular}{|c|c|c|c|c|}
\hline 98 & C. toulou & 2nd & $\bar{C}$ & $\begin{array}{l}\text { Kunming, Yuxi, Simao, Baoshan, Honghe, Xishuangbanna, } \\
\text { Wenshan }\end{array}$ \\
\hline 99 & Tyto alba & 2nd & $\mathbf{R}$ & Kunming, Simao, Xishuangbanna \\
\hline 100 & T. capensis & 2nd & $\mathrm{C}$ & Kunming, Simao, Xishuangbanna, Honghe \\
\hline 101 & Phodilus badius & 2nd & $\mathrm{E}$ & Xishuangbanna \\
\hline 102 & Otus spilocephalus & 2nd & $\mathbf{R}$ & Simao, Lincang \\
\hline 103 & O. scops & 2nd & $\mathbf{R}$ & Yuxi, Honghe, Dali, Nujiang, Xishuangbanna \\
\hline 104 & O. bakkamoena & 2nd & $\mathbf{R}$ & Kunming, Simao, Xishuangbanna, Honghe, Dehong \\
\hline 105 & Bubo nipalensis & 2nd & $\mathbf{R}$ & Honghe \\
\hline 106 & B. bubo & 2nd & C & All parts of Yunnan \\
\hline 107 & Ketupa zeylonensis & 2nd & $\mathbf{R}$ & Kunming, Honghe, Xishuangbanna, Baoshan \\
\hline 108 & K. flavipes & 2nd & $\mathbf{R}$ & Xishuangbanna, Zhaotong \\
\hline 109 & K. ketupu & 2nd & $\mathbf{E x}$ & unknown \\
\hline 110 & Glaucidium brodiei & 2 nd & $\mathrm{C}$ & $\begin{array}{l}\text { Simao, Xıshuangbanna, Lincang, Yuxi, Honghe, Dali, Chuxiong, } \\
\text { Nujiang }\end{array}$ \\
\hline 111 & G. cuculoides & 2nd & $\mathrm{C}$ & All parts of Yunnan \\
\hline 112 & Ninax scutulata & 2nd & $\mathbf{R}$ & Xishuangbanna, Honghe, Simao, Lincang, Baoshan \\
\hline 113 & Strix leptogrammica & 2nd & $\mathbf{R}$ & Xishuangbanna, Dehong, Baoshan \\
\hline 114 & S. aluco & 2nd & $\mathbf{R}$ & Kunming, Baoshan, Diqing, Lijiang, Zhaotong \\
\hline 115 & Asio flammeus & 2nd & $\mathbf{R}$ & Simao, Xishuangbanna, Baoshan \\
\hline 116 & Hemiprocne Longipennis & 2nd & $\mathbf{R}$ & Lincang, Xishuangbanna \\
\hline 117 & Harpactes oreskios & 2nd & $\mathbf{R}$ & Xishuangbanna, Lincang, Baoshan \\
\hline 118 & Alcedo meninting & 2nd & $\mathbf{R}$ & Xishuangbanna \\
\hline 119 & Pelargopsis capensis & 2nd & $\mathbf{R}$ & Xishuangbanna \\
\hline 120 & Merops leschenaulti & 2nd & $\mathrm{C}$ & $\begin{array}{l}\text { Xishuangbanna, Simao, Yuxi, Honghe, Dehong, Lincang, } \\
\text { Nujiang }\end{array}$ \\
\hline 121 & M. orientalis & 2nd & $\mathbf{R}$ & Dehong, Simao, Xishuangbanna, Chuxiong, Yuxi \\
\hline 122 & Ptilolaemus tickelli & 2nd & $\mathbf{R}$ & Xishuangbanna \\
\hline 123 & Aceros nipalensis & 2nd & $\mathbf{R}$ & Xishuangbanna, Honghe \\
\hline 124 & Anthracaceros coronatus & 2nd & $\mathbf{R}$ & Dehong, Lincang, Simao, Xishuangbanna \\
\hline 125 & Buceros bicornis & 2nd & $\mathbf{R}$ & Dehong, Lincang, Xishuangbanna \\
\hline 126 & Dryocopus javensis & 2nd & $\mathbf{R}$ & Simao, Lijiang, Xishuangbanna, Nujiang \\
\hline 127 & Serilophus lunatus & 2nd & $\mathbf{R}$ & Xishuangbanna, Honghe, Lincang \\
\hline 128 & Psarisomus dalhousiae & $2 n d$ & $\mathrm{C}$ & Xishuangbanna, Honghe, Lincang, Dehong, Wenshan, Simao \\
\hline 129 & Pitta nipalensis & 2nd & $\mathbf{R}$ & Xishuangbanna, Honghe \\
\hline 130 & P. soror & 2nd & $\mathbf{R}$ & Honghe \\
\hline 131 & P. cyanea & 2nd & $\mathbf{R}$ & Xishuangbanna \\
\hline 132 & P. nympha & 2nd & $\mathbf{R}$ & Wenshan \\
\hline 133 & P. brachyuara moluccensis & 2nd & $\mathbf{R}$ & Xishuangbanna \\
\hline 134 & P. sordida & 2nd & $\mathbf{R}$ & Xishuangbanna, Honghe \\
\hline 135 & $P$. oatesi & 2nd & $\mathrm{R}$ & Xishuangbanna, Simao, Dehong, Honghe, Wenshan \\
\hline 136 & P. phayrei & 2nd & $\mathbf{R}$ & Xishuangbanna \\
\hline
\end{tabular}

LP. Impartant Levels in Protection in China: 1at: First-ranked protected species in China; 2nd: Second-ranked protected species in China: Bx (Extinet); Species were only recorded before 1926 and not found since then: E (Endangered): Species had a small population before 1965 and almost were nor found since then: V (Vulnerabte): Species only have a few records since 1970, or species have a very narrow range and their habitats were destructed seriously; R (Rare): Species have a narrow range. and/or definitely have a small population in Yunnan and found many times in recent years: C (Common): Species have a wide range, and have a large population and were not difficult to be found in recent years. 